\title{
Optical Fibre Current Sensor for Electric Power Industry
}

\author{
K. Barczak ${ }^{a, *}$, T. Pustelny ${ }^{a}$, D. Dorosz ${ }^{b}$ And J. Dorosz ${ }^{b}$ \\ ${ }^{a}$ Department of Optoelectronics, Silesian University of Technology, Krzywoustego 2, 44-100 Gliwice, Poland \\ ${ }^{b}$ Department of Optical Radiation, Białystok Technical University, Wiejska 45, 15-950 Białystok, Poland \\ The paper deals with the optical fibre current sensor based on new optical fibre made of glass with a high \\ value of refractive index. This kind of glass was developed to meet the requirements of these optical fibres. The \\ aim of investigations was to develop a new optical fibre with adequate magneto-optical properties and a relatively \\ weak linear birefringence resulting from the process of its production. These features are of essential importance \\ for optical fibre current sensors. The results of investigations concerning such optical fibres have been presented, \\ displaying satisfactory magneto-optical properties, as well as only low linear birefringence induced by stresses \\ arising in the course of their manufacturing.
}

PACS numbers: 42.70.-a, 42.81.-i, 42.79.Pw, 78.20.Ls

\section{Introduction}

Nowadays, in the age of the development of metrology, when new kinds of sensors and new alternatives of applying already existing sensors have come into being, also sensors making use of magneto-optical phenomena have attained importance. They are counterparts of traditional sensors of magnetic fields and electric currents, presently used to a large extent in power electrical engineering. These are mainly the so-called current transformers and sensors of magnetic fields inside the mechanisms and electric power machines, such as transformers and electrical engines. All the traditional solutions are based on the phenomenon of electromagnetic induction as well as on the Hall effect, i.e. on measurements of the electrical voltage or current.

Optical sensors of the magnetic field make use of magneto-optical phenomena, and information about the measured quantity is concealed in the parameters of the light beam propagating in the optical medium [1-5]. Solutions of this kind are of enormous interest for those dealing with electrical power metrology, because due to their non-electricity they are absolutely safe, permit to reduce considerably the dimensions of the sensor, need not be cooled down and are not subjected to electromagnetic disturbances $[6,7]$.

The dielectricity of optic media permits to apply such sensors inside electrical power machines while these are operating [8]. At present most effectively so-called optical fibre current sensors (OFCSs) consisting of optical fibres are being developed (Fig. 1). This solution is based on the Faraday effect, which is the most often applied magneto-optical effect in this kind of sensors [3, 4].

In the present paper the authors focused their atten-

* corresponding author; e-mail: kamil.barczak@polsl.pl

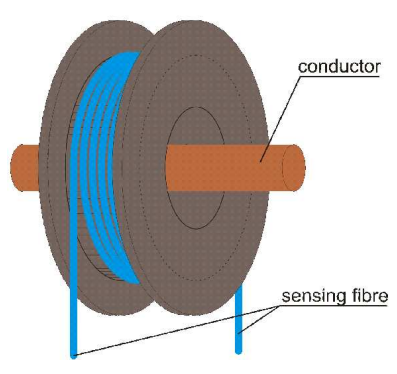

Fig. 1. Image of the structure of OFCS.

tion on the search for new special kinds of optical fibres which might be applied in optical current fibre sensors characterized by a high sensitivity and small susceptibility to deformation $[9,10]$. In result of these researches and analyses a new material has been suggested displaying a high refractive index and can be used to produce single mode step-index fibres [11]. Fibres made of such glass were subjected to extensive investigations. The present paper provides the results of investigations and determined parameters of these glasses, as well as a description of the developed sensor of electric currents.

\section{High refractive index special optical fibres}

A matrix of silicon dioxide (silica) glass was used, modified with the following oxides: $\mathrm{PbO}, \mathrm{Al}_{2} \mathrm{O}_{3}, \mathrm{~B}_{2} \mathrm{O}_{3}$, $\mathrm{Na}_{2} \mathrm{O}$, and $\mathrm{K}_{2} \mathrm{O}$. The concentrations of the respective components in the glass are contained within the following ranges: $\mathrm{SiO}_{2} \quad(55-70 \mathrm{~mol} \%), \quad \mathrm{PbO}$ (8-20 mol\%), $\mathrm{Al}_{2} \mathrm{O}_{3}$ (6-10 mol\%), $\mathrm{B}_{2} \mathrm{O}_{3}$ (1-5 mol\%), $\mathrm{Na}_{2} \mathrm{O}$ (5-8 mol\%), $\mathrm{K}_{2} \mathrm{O}$ (4-7 mol\%).

In order to produce a single-mode fibre the crucible method was applied. The core and cover of the fibre consist of this glass with the values of refractive indices of 
light: $n_{\mathrm{r}}=1.6195$ and $n_{\mathrm{p}}=1.6190$, respectively. The cover of the fibre was developed basing on the modified composition of the glass core, changing the proportion of $\mathrm{PbO} / \mathrm{Al}_{2} \mathrm{O}_{3}$, in order to control the value of its refractive index. The core glass and the glass of the cover displayed similar technological properties, viz. viscosity and the coefficient of thermal expansion, ensuring minimum internal stresses in the optical fibres. The spectrum of transmission of the glass was determined in the range 380-1100 nm (Fig. 2). The level of transmission of the produced glass is of the order $95 \%$. At the value of the microhardness amounting to $9 \mathrm{GPa}$ this permits to use this material for the production of waveguides.

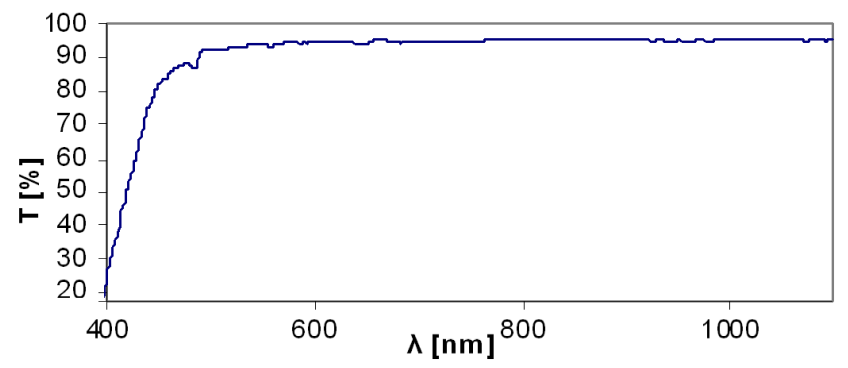

Fig. 2. Spectrum of transmission of the glass (thickness of the sample $2 \mathrm{~mm}$ ).

Besides the required optical properties, waveguides applied as sensors must be characterized by extremely low mechanical stresses affecting considerably their propagating properties. Additional requirements concern the thermal parameters of the applied glass. The first condition is resistance of the glass to crystallization, determined basing on the thermal differential analysis. The obtained glass did not display any tendency to crystallization, which fact is of crucial importance in the thermal treatment of the glass during manufacturing the optical fibre.

The elaborated system of the glasses presented above was applied to produce single-mode fibres (at $633 \mathrm{~nm}$ ) with a core refractive index amounting 1.62. The necessity of satisfying the condition of single-modality at an assumed large diameter of the core $(\approx 12 \mu \mathrm{m})$ required a strict control of the difference between the refractive indices of core glass and cover glass, which amounted to $\Delta n=0.0005$. As it was admitted, for the manufacturing of a single-mode optical fibre the crucible method was used. The process was performed conventionally according to standard at a viscosity of the glass $10^{4}-10^{5} \mathrm{dPa} \mathrm{s}$, at a temperature $910^{\circ} \mathrm{C}[12,13]$. The attenuation of the produced optical fibre, measured by means of the cutting-off method [14] at a wavelength of $633 \mathrm{~nm}$ amounts to $0.1 \mathrm{~dB} / \mathrm{m}$.

\section{Optical fibre current sensor}

The produced optical fibres are to be applied in the system of a fibre current sensor. The complete system is to be seen in Fig. 3. The system is characterized by the simplicity of structure. The output signal can be easily recalculated in the value of the current flowing through the conductor. The practical realization of the sensor head for laboratory experiments has been illustrated in Fig. 4. The diameter of the conductor is $40 \mathrm{~mm}$, and the diameter of the reel (on which the optical fibre was coiled) $100 \mathrm{~mm}$. At a current intensity $1 \mathrm{kA}$ the induction of the magnet field along the optical fibre amounts to $4 \mathrm{mT}$.

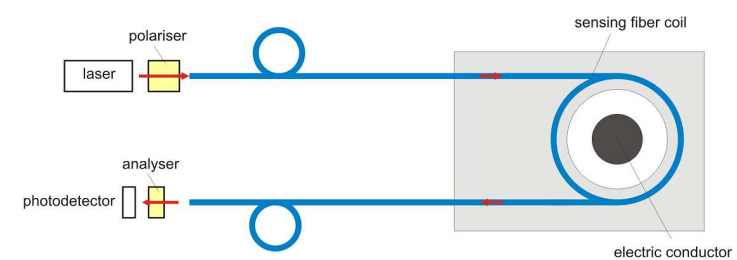

Fig. 3. Diagram of the structure of the optical fibre current sensor.

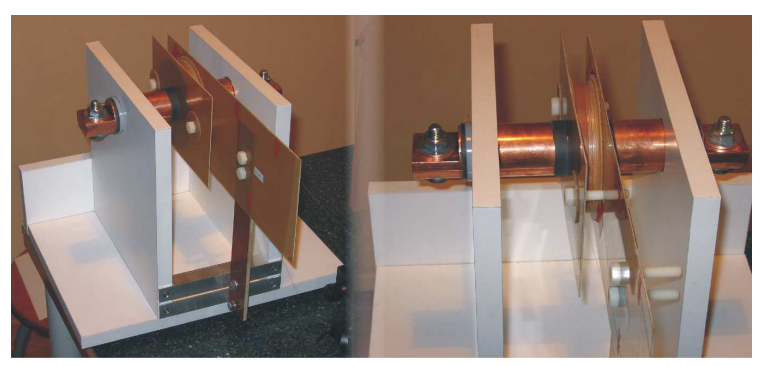

Fig. 4. Photos of the developed test model of the head of the waveguide current sensor.

The first stage of investigations consisted of magneto-optic properties of the aforesaid glass. The Verdet constant for the wavelength $635 \mathrm{~nm}$ was $6.58 \mathrm{rad} /(\mathrm{T} \mathrm{m})$; this result confirmed the good magneto-optical properties of the obtained glass.

The next stage comprised tests of the produced optical fibres in the magnetic field. The aim of these investigations was to determine the magneto-optical properties. For this purpose the tested optical fibre was placed inside an air-core coil, $80 \mathrm{~cm}$ long, supplied with direct current. A series of measurements were carried out concerning various values of azimuth of linear input polarization $\phi$. For each value of azimuth of linear input polarization $\phi$ the state of polarization was recorded at the output for the value of induction of the magnetic field $B=0 \mathrm{mT}$ and the value $B=35 \mathrm{mT}$. Figure 5 presents the values of changes of the azimuth of the state of polarization depending on the azimuth of linearly input polarization $\phi$. The values of the changes of the azimuth of the state of output polarization $\Delta \alpha$ permit to determine the Verdet constant. The range of changes of this constant (Fig. 5) reaches from $0.29 \mathrm{rad} /(\mathrm{T} \mathrm{m})$ to $5.93 \mathrm{rad} /(\mathrm{T} \mathrm{m})$. Figure 6 presents the value of changes of the angle of ellipticity of the output polarization $\Delta \gamma$ on the azimuth of the linearly input light polarization $\phi$. 


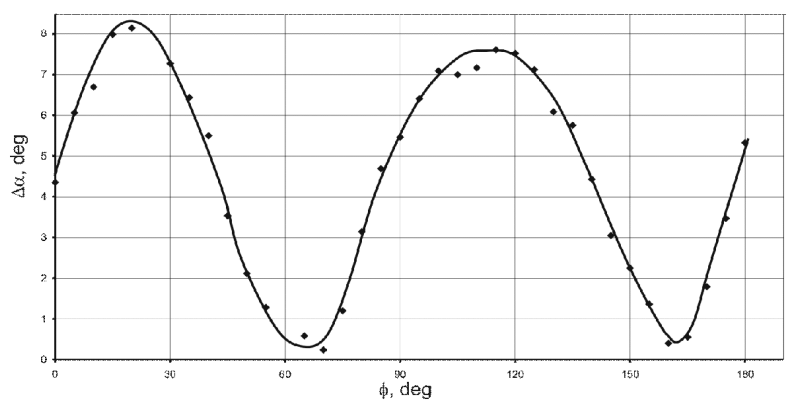

Fig. 5. Changes of the azimuth of the state of output polarization $\Delta \alpha$ as a function of the azimuth of input polarization $\phi$.

The obtained results indicate that the magneto-optical effect in elaborated fiber depends on the angle of the azimuth of input polarization. It is also to be noted that the ellipticity of polarization undergoes changes in the magnetic field. The maximum of these changes occurs in the vicinity of the minimum changes of the azimuth of output polarization $\Delta \alpha$. This means that the medium in which the light propagates is a linearly birefringent medium. This is undesirable effect connected with stresses arising in the fibre in the course of its production and due to the deformation of the optical fibre. The diagrams presented in Figs. 5 and 6 determine the point of operation of this sensor, and thus also the optimal choice of the azimuth of input linear polarization.

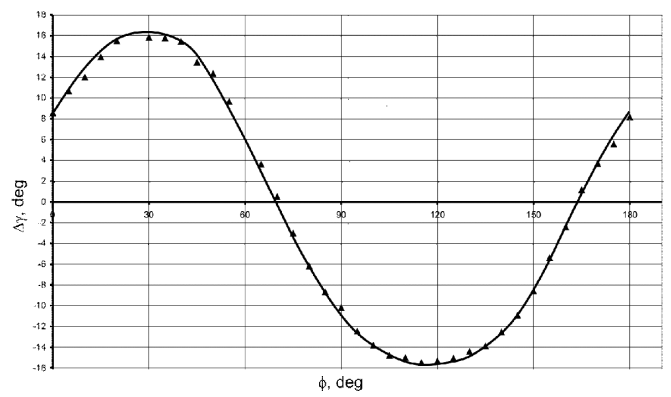

Fig. 6. Changes of the angles of ellipticity of output polarization $\Delta \gamma$ as a function of the azimuth of input polarization $\phi$.

\section{Conclusions}

The performed investigations indicate distinctly that the presented optical fibre based on glass with a high refractive index is characterized by a high value of the Verdet constant. It proved to be impossible, however, to eliminate in this structure the linear birefringence indicated by the stresses. The results of measurements presented in Figs. 5 and 6 illustrate the influence of the azimuth of input linear polarization in elaborated optical fibre on the change of state of the polarization of light for the magneto-optical effect. Values of these changes are similar in order to those occurring in a birefringent medium. Hence, the birefringence occurring in the course of production of this optical fibre is not accidental and does not change in time. From the point of view of applying these optical fibres in optical fibres current sensors (OFCSs) are promising.

The performed investigations permitted also to produce interesting kinds of glass with good magneto-optical properties, which inspired the authors to take up tests concerning optical fibre current sensors with internal conversion.

\section{Acknowledgments}

The work was sponsored by the State Committee for Scientific Research within the grant No. N515 409534.

\section{References}

[1] T. Pustelny, Physical and Technical Aspects of Optoelectronics Sensors, Wydawnictwo Politechniki Śląskiej, Gliwice 2005.

[2] S. Pustelny, A. Wojciechowski, M. Gring, M. Kotyrba, J. Zachorowski, W. Gawlik, J. Appl. Phys. 103, 063108 (2008).

[3] W. Gawlik, L. Krzemien, S. Pustelny, D. Sangla, J. Zachorowski, M. Graf, A.O. Sushkov, D. Budker, Appl. Phys. Lett. 88, 131108 (2006).

[4] M.P. Ledbetter, V.M. Acosta, S.M. Rochester, D. Budker, S. Pustelny, Phys. Rev. A 75, 023405 (2007).

[5] T. Pustelny, M. Grabka, Acta Phys. Pol. A 116, 385 (2009).

[6] B. Lee, Opt. Fiber Technol. 9, 57 (2003).

[7] J.D.P. Hrabliuk, in: IEEE PES Winter Power Meeting, Vancouver, Eds. J. Paserba, G. Anderson, University of Vancouver, Vancouver (British Columbia, Canada) 2002, p. 341.

[8] K. Barczak, T. Pustelny, Z. Zycki, T. Blazejczyk, Acta Phys. Pol. A 116, 250 (2009).

[9] A.M. Smith, Appl. Opt. 19, 2606 (1980).

[10] K. Barczak, T. Pustelny, D. Dorosz, J. Dorosz, Europ. Phys. J. Spec. Topics 154, 11 (2008).

[11] K. Barczak, T. Pustelny, D. Dorosz, J. Dorosz, Acta Phys. Pol. A 116, 247 (2009).

[12] J. Dorosz, Polski Biuletyn Ceramiczny 86, 115 (2005) (in Polish).

[13] D. Dorosz, M. Kochanowicz, J. Dorosz, Acta Phys. Pol. A 116, 298 (2009).

[14] D. Dorosz, K. Barczak, T. Pustelny, J. Dorosz, Acta Phys. Pol. A 114, A-61 (2008). 